Original Research Paper

\title{
Environmental Protection through Nuclear Energy
}

\author{
${ }^{1}$ Florian Ion T. Petrescu, ${ }^{2}$ Antonio Apicella, ${ }^{3}$ Relly Victoria V. Petrescu, ${ }^{4}$ Samuel P. Kozaitis, ${ }^{5}$ Ronald B. \\ Bucinell, ${ }^{6}$ Raffaella Aversa and ${ }^{7}$ Taher M. Abu-Lebdeh
}

\author{
${ }^{1}$ Department of Theory of Mechanisms and Robots, Bucharest Polytechnic University, Bucharest, Romania \\ ${ }^{2}$ Advanced Materials Lab, Second University of Naples, Naples, Italy \\ ${ }^{3}$ Romanian Society of Robotics, Bucharest branch, Polytechnic University of Bucharest, Bucharest, Romania \\ ${ }^{4}$ General Motors Research Laboratory, \\ Florida Institute of Technology, Electrical and Computer Engineering, Florida, United States \\ ${ }^{5}$ Department of Mechanical Engineering, \\ American Society of Mechanical Engineers (ASME), Union College, NY, United States \\ ${ }^{6}$ Advanced Materials Lab, Second University of Naples, Naples, Italy \\ ${ }^{7}$ North Carolina A and T State Univesity, United States
}

Article history

Received: 19-09-2016

Revised: 27-09-2016

Accepted: 28-09-2016

Corresponding Author:

Florian Ion T. Petrescu

Department of Theory of

Mechanisms and Robots,

Bucharest Polytechnic

University, Bucharest, Romania

Email: petrescuflorian@yahoo.com

\begin{abstract}
Environmental protection through implementation of green energies is progressively becoming a daily reality. Numerous sources of green energy were introduced in recent years. Although this process initially started with difficulties, it finally resulted in an acceleration and implementation of new green energy technologies. Nonetheless, new major obstacles are emerging. The most worldwide difficult obstacle encountered, especially for wind and photovoltaic electric power plants, is the not regular and predictable green energy production. This study proposes solutions designed to solve this unpleasant aspect of irregular production of green energy. The basic idea refers to the construction of specially designed nuclear power plants acting as energy buffers. Nuclear power plants, indeed, may behave as proper energy buffers able to work to a minimum capacity when the green energy (i.e., wind power or PV) is steadily produced (namely, when the energy generated by the turbines or PV panels is at full constant capacity) but that can also run at progressively increased capacities when the wind or solar energy production reduces or stops. The work get two major contributions: 1-propose to the achievement of an energy buffer using nuclear power plants (for the moment on nuclear fission); 2-shows some theoretical aspects important needed to carry out the reaction of the fusion.
\end{abstract}

Keywords: Environmental Protection, Green Energy, Nuclear Energy, Nuclear Fusion, Renewable Energy

\section{Introduction}

Our planet anthropogenic and natural carbon dioxide $\left(\mathrm{CO}_{2}\right)$ gas is released every day into the earth's atmosphere and it is able to last for the next 100 years. Carbon dioxide, which is the principal greenhouse gas emitted by anthropical activities, is naturally present in the atmosphere as part of the Earth's carbon cycle that has been altering by human activities impairing the ability of natural $\mathrm{CO}_{2}$ sinks to remove this gas from the atmosphere (Le Quéré et al., 2015). Yearly global carbon (C) emissions from fossil fuel use were near to 10.000 gigatonnes (wich correspond to 36.700 gigatonnes of
Carbon dioxide per year) in the last years and it is steadily increasing at a rate of $1 \%$ year (Peters et al., 2012).

This increased content of carbon dioxide favors global warming of our planet. One answer to global warming is to exchange and retrofit current technologies with carbon dioxide free alternatives that have comparable or even higher performance.

A serious crisis of the energy resources has characterized the years 1970-1980. Hydrocarbon-based energies were polluting while increasingly exhausting. Fossil fuels alimented vehicles and big industries (large energy consumers) number proliferated continuously. It was then urgently needed to develop new energy 
resources. Fission nuclear energy was introduced in these dramatic scenarios as a necessary evil. Nuclear fission power plants have secured a new large amount of energy needed by our blue planet (Petrescu and Petrescu, 2011).

These nuclear plants present great advantages but also several disadvantages: Nuclear fission energy has been managed to take over the existing energy deficit and to provide more time to the major oil companies to discover new deposits of oil, natural and shale gas. Moreover, under controlled conditions, nuclear fission energy is generally cheap and safe. However, even though the fission nuclear energy uses a fuel (Uranium) that exists in large quantities on the planet, it begins to run low as already occurring for hydrocarbons. Moreover, the thorniest issue at the nuclear fission plant remains that both the fuel used (enriched Uranium) and the exhausted byproducts are radioactive and dangerous.

Nuclear fission energy was then fundamentally a needed but hardly tolerated evil. Despite all related risks, the use of this type of energy is managing the increasing critical energy crisis of humanity until new advanced technologies will allow us the transition to alternative cleaner energies.

Nuclear fusion energy, once further implemented, could be the most powerful energy source for mankind. Although great advances have been made in this direction, the nuclear fusion power plants have not yet realized. Nuclear fusion power could not yet be made, but their season is fast approaching. The advantages of nuclear fusion energy are enormous.

Primarily, the fuel used in this technology (Hydrogen or water) is not radioactive. Of course this is not the first isotope of hydrogen or normal water, as a fusion reaction between two protons is made extremely difficult (only at high temperatures, in stars). Usually it uses the second isotope of hydrogen (Deuterium, which is the nucleus with one proton and one neutron) or heavy water (a molecule containing an atom of oxygen and two atoms of Deuterium). Water is found everywhere, so the fuel needed for fusion reaction is infinite, cheap, easy to find, friendly and non-toxic or radioactive. The technology for producing heavy water from water today is well planned.

The products resulting from fusion reactions are a large amount of energy and helium (an inert gas), so without radioactive wastes (such as to the nuclear fission). The reaction itself is much easier to control (De Ninno et al., 2002).

Since it is not predictable when fusion plants will be operative in large quantity, it is compelling to equip us in advance with green energy farms. Environmental protection through the implementation of green energy is progressively becoming a daily reality. Various sources of green energy were introduced, especially in recent years, in the entire planet. The process, which started hard but finally resulted in the acceleration and implementation of new green energy sources, is still impaired by major emerging hindrances. The most difficult obstacle experienced in worldwide, was the unpredictable and fluctuating green energy production. All new energies need to have no disagreeable consequences such as those occurred for the fossil fuels or the nuclear energy. Valid planetary alternative energy sources need to be renewable and are thought to be "free" energy sources. These sources need to have reduced carbon emissions, compared to conventional energy sources. It may include: Biomass, Wind, Photovoltaic Solar, Geothermal, Hydroelectric, Tidal, Wave, or Nuclear, (Petrescu and Petrescu, 2015).

The most presently numerous ones, since they are easy to be built and run, are the wind and the solar photovoltaic farms. But their great reliability and technical problem is to have phases when they produce less, or do not produce at all.

The basic idea of this paper refers to the construction of nuclear power plants specially designed to represent a factual energy buffer. These specially designed nuclear power plants may become effective energetic buffer, able to work to a minimum capacity when the green wind or PV solar power energy is regularly produced (namely, when the energy generated by the wind turbines or PV panels is running at full capacity) but that can also run at progressively increased capacities when the wind or solar energy production reduces or stops.

\section{Materials and Methods}

Current fission nuclear power only represents a transition to the thermonuclear energy based on light nuclei fusion.

The main particularity of fusion reaction is the prevalence of the fuel used (Deuterium). It can be obtained very simply from ordinary water. Harold Urey has extracted Deuterium from water for the first time in 1931. Even at that time some small electrostatic linear accelerators have been indicated the fact that D-D reaction (the fusion between two Deuterium nuclei) was an exothermic reaction. Today we already know that not only the second isotope of hydrogen-Deuterium (a Deuterium nucleus contains two nucleons, one proton and one neutron) produces fusion energy, but also the third (heavier) isotope of hydrogen-Tritium (a Tritium nucleus contains three nucleons, one proton and two neutrons) can produce energy by nuclear fusion.

The fusion reaction would be even and simple if we use still heavier isotopes of hydrogen, however, these isotopes can't be easily obtained today. Even the third isotope of hydrogen, Tritium can't be obtained directly (as Deuterium) but only by nuclear reactions between two Deuterium nuclei. The only first reaction is possible just between two nuclei of Deuterium, from which can be obtained either (a) Tritium nucleus plus a proton and energy, or an isotope of helium with a neutron and energy (see relationships 1-2), (Petrescu, 2012): 


$$
\begin{aligned}
& { }_{1}^{2} \mathrm{D}+{ }_{1}^{2} \mathrm{D}->_{1}^{3} \mathrm{~T}+1 \mathrm{MeV} \\
& { }_{1}^{1} \mathrm{H}+3 \mathrm{MeV}={ }_{1}^{3} \mathrm{~T}+{ }_{1}^{1} \mathrm{H}+4 \mathrm{MeV} \\
& { }_{1}^{2} \mathrm{D}+{ }_{1}^{2} \mathrm{D}->_{2}{ }^{3} \mathrm{He}+0.8 \mathrm{MeV} \\
& +{ }^{1} \mathrm{n}+2.5 \mathrm{MeV}={ }_{2}^{3} \mathrm{He}+{ }^{1} \mathrm{n}+3.3 \mathrm{MeV}
\end{aligned}
$$

Once the Tritium has been obtained, the fusion between a nucleus of Deuterium and one of Tritium can occur (see the Equation 3), from which it can be obtained an atom of Helium with a neutron and high energy. Such a fusion reaction is preferable but to obtain it we need first a reaction between two Deuterium nuclei:

${ }_{1}^{2} \mathrm{D}+{ }_{1}^{3} \mathrm{~T}->{ }_{2}^{4} \mathrm{He}+3.5 \mathrm{MeV}$

$+{ }^{1} n+14 \mathrm{MeV}={ }_{2}^{4} \mathrm{He}+{ }^{1} n+17.5 \mathrm{MeV}$

A nucleus of Deuterium may produce another nuclear reaction between a nucleus of Deuterium and an isotope of Helium (see the Equation 4):

$$
\begin{aligned}
& { }_{1}^{2} \mathrm{D}+{ }_{2}^{3} \mathrm{He}>{ }_{2}^{4} \mathrm{He}+3.7 \mathrm{MeV} \\
& { }_{1}{ }_{1}^{1} \mathrm{H}+14.7 \mathrm{MeV}={ }_{2}^{4} \mathrm{He}+{ }_{1}{ }_{1} \mathrm{H}+18.4 \mathrm{MeV}
\end{aligned}
$$

To make these reactions occurring, it should ensure that the Deuterium nuclei have enough kinetic energy to overcome the electrostatic rejection forces due to the positive charges of protons from the nuclei (Petrescu and Petrescu, 2014).

Deuterium fuel is delivered in heavy water, $\mathrm{D}_{2} \mathrm{O}$.

To obtain Tritium we need first of a reaction between two Deuterium nuclei.

Tritium may be obtained in the laboratory and by the following reaction (Equation 5):

${ }_{3}^{6} \mathrm{Li}+{ }^{1} \mathrm{n}-->_{1}{ }^{3} \mathrm{~T}+{ }_{2}{ }^{4} \mathrm{He}+4.6 \mathrm{MeV}$

The third element in Mendeleev's table (Lithium) is found in nature in sufficient quantities. Neutrons needed to produce the reaction 5 (with Lithium), develop from the second and from the first + third reaction. This means that also Deuterium (heavy water) has to be added to Lithium.

Raw materials to start fusion are, hence, Deuterium and Lithium. All shown fusion reactions finally generate energy and $\mathrm{He}$ that is recognized to act as an inert element. Because of this, fusion reaction is clean and far superior to nuclear fission.

Hot fusion spontaneously occurs at very high temperatures. Getting the necessary high temperature for hot fusion is still difficult and for that reason we must focus now on cold nuclear fusion. In order to induce cold fusion we need to accelerate the Deuterium nuclei in linear or circular accelerators. The proper energy of accelerated Deuterium nuclei should be well calibrated for a positive final yield of fusion reactions (to induce more nuclei merging than fission).

Electromagnetic fields needed to maintain the plasma (cold or warm), should be held (especially at cold fusion) to constrain more closely the nuclei.

We need to blast the fuel with accelerated Deuterium nuclei. The fuel will be made from heavy water and Lithium. The optimal proportion of Lithium is to be tested. In order to achieve a strong ionization of the fuel, it is compelling to keep fuel in the plasma state. In these conditions, instead of Deuterium atoms, Deuterium nuclei (positive ions) are produced, which can be accelerated by the electromagnetic fields.

\section{Results and Discussion}

Presented work shows and some theoretical aspects important needed to carry out the reaction of the fusion. For a better understanding of these new aspects it is necessary to be investigated and the work (Petrescu and Calautit, 2016).

The smallest radius between Deuterium and Tritium is the radius of Deuterium nucleus (Equation 6), (Halliday and Robert, 1966):

$$
\begin{aligned}
& \text { Deuterium } A=2 A^{1 / 3}=1.259921 \\
& -->R_{D}=1.8268855223476 E^{-15}[\mathrm{~m}]
\end{aligned}
$$

Tritium $A=3 A^{1 / 3}=1.44224957$

$->R_{T}=2.0912618769457 E^{-15}[\mathrm{~m}]$

Now we must calculate the minimum distance between two particles that need to gather together. This distance is just the Deuterium nucleus diameter, $d_{12 D}$ (Equation 7):

$$
\begin{aligned}
& d_{12 D}=2 R_{D}=2 x 1.8268855223476 E^{-15}[\mathrm{~m}] \\
& =3.6537710446952 E^{-15}[\mathrm{~m}]=3.653771 E^{-15}[\mathrm{~m}]
\end{aligned}
$$

The energy potential that rejects two particles each other can be obtained from the following expression (8), (Halliday and Robert, 1966):

$$
\begin{aligned}
& U=E_{p}=q_{1} q_{2} /\left(4 \pi \varepsilon_{0} d_{12}\right) \\
& =\left(1.602 E^{-19}\right)^{2} /\left(4 \pi 8.8541853 E^{-12} \times 33.653771 E^{-15}\right) \\
& =6.3128464855 E^{-14}[\mathrm{~J}]=6.3128464855 E^{-14} \times 6.242 E^{18}[\mathrm{eV}] \\
& =3.94 E^{5}[\mathrm{eV}]=3.94 E^{2}[\mathrm{keV}]=394[\mathrm{keV}]
\end{aligned}
$$

This is the expression of the potential energy between two adjacent particles (electrostatic potential energy), which should be the energy with that a 
particle needs to be accelerated before collision (Equation 8 or 9, Fig. 1), This electrostatic potential energy must to be the same with the (final) kinetic energy of motion translational of the accelerated particle $E_{p}=1 / 2 \mathrm{mv}^{2}$ :

$\left\{\begin{array}{l}U=E_{p}=\frac{1}{4 \pi \cdot \varepsilon_{0}} \cdot \frac{q_{1} \cdot q_{2}}{d_{12}}=\frac{q_{1} \cdot q_{2}}{8 \pi \cdot \varepsilon_{0} \cdot R} \\ E_{p}=\frac{1}{2} m \cdot v^{2}\end{array}\right.$

The radius of Deuterium at rest (without motion, static), was determined in Fig. 1 according to the following relationships (6a) and (10), (Petrescu and Calautit, 2016):

$\left\{\begin{array}{l}R_{D}=r_{0} \cdot A^{1 / 3} \\ r_{0}=1,45 E-15[\mathrm{~m}] \text { the average radius } \\ \text { of a nucleon fixed } \\ \mathrm{A}=\text { the atomic mass }\end{array}\right.$

One obtains first the needed speed value (v) of the accelerated particle required for fusion (Equation 11), (Petrescu and Calautit, 2016):

$v=691664.8602[\mathrm{~m} / \mathrm{s}]$

With expression 12 it may determine now with high accuracy the radius of a Deuteron or any other elementary moving particle, as a function of its velocity, $v$ and its rest mass, $m_{0}$, (Petrescu and Calautit, 2016).

$R=\sqrt{\frac{10}{8}} \cdot \frac{h \cdot \sqrt{c^{2}-v^{2}} \cdot \sqrt{c^{2}-\frac{v^{2}}{2}-c \cdot \sqrt{c^{2}-v^{2}}}}{\pi \cdot m_{0} \cdot c^{2} \cdot v}$

With $m_{0}$ deuteron $=3.34524 \mathrm{E}-27[\mathrm{~kg}]$ and $v=$ $691664.8602[\mathrm{~m} / \mathrm{s}]$, the radius of a Deuteron is $R_{D}=$ 1.91788E-19 [m], with $h=>$ the Planck constant, $h=$ $6.626 \mathrm{E}-34[\mathrm{Js}]$ and $c=$ the light velocity, $c=$ $2.997925 \mathrm{E} 8[\mathrm{~m} / \mathrm{s}]$.

Potential energy of a Deuteron in movement has the value reported below (Equation 13):

$$
\begin{aligned}
& U=E p=6.01333 E-10[\mathrm{~J}] \\
& =3753521838[\mathrm{eV}]=3753521.838[\mathrm{KeV}]= \\
& =3753.521838[\mathrm{MeV}]=3.753521838[\mathrm{GeV}]
\end{aligned}
$$

Nuclear fission energy has been managed to take over the existing energy deficit and to provide more time to the major oil companies to discover new deposits of oil, natural and shale gas. Moreover, under controlled conditions, nuclear fission energy is generally cheap and safe.

$$
\begin{aligned}
& d_{12 D}=2 \cdot R_{D}=2 \cdot 1.8268855223476 \cdot 10^{-15}[\mathrm{~m}]= \\
& =3.6537710446952 \cdot 10^{-15}[\mathrm{~m}]= \\
& \approx 3.653771 \cdot 10^{-15}[\mathrm{~m}]
\end{aligned}
$$

Fig. 1. Two adjacent particles of Deuterium

However, even though the fission nuclear energy uses a fuel (Uranium) that exists in large quantities on the planet, it begins to run low as already occurring for hydrocarbons. Moreover, the thorniest issue at the nuclear fission plant remains that both the fuel used (enriched Uranium) and the exhausted byproducts are radioactive and dangerous.

Nuclear fission energy was then fundamentally a needed but hardly tolerated evil. Despite all related risks, the use of this type of energy is managing the increasing critical energy crisis of humanity until new advanced technologies will allow us the transition to alternative cleaner energies.

Nuclear fusion energy, once further implemented, could be the most powerful energy source for mankind. Although great advances have been made in this direction, the nuclear fusion power plants have not yet realized. Nuclear fusion power could not yet be made, but their season is fast approaching. The advantages of nuclear fusion energy are enormous.

Primarily, the fuel used in this technology (Hydrogen or water) is not radioactive. Of course this is not the first isotope of hydrogen or normal water, as a fusion reaction between two protons is made extremely difficult (only at high temperatures, in stars). Usually it uses the second isotope of hydrogen (Deuterium, which is the nucleus with one proton and one neutron) or heavy water (a molecule containing an atom of oxygen and two atoms of Deuterium). Water is found everywhere, so the fuel needed for fusion reaction is infinite, cheap, easy to find, friendly and non-toxic or radioactive. The technology for producing heavy water from water today is well planned.

The third element in Mendeleev's table (Lithium) is found in nature in sufficient quantities. Neutrons needed to produce the reaction 5 (with Lithium), develop from the second and from the first + third reaction. This means that also Deuterium (heavy water) has to be added to Lithium.

Raw materials to start fusion are, hence, Deuterium and Lithium. All shown fusion reactions finally generate energy and $\mathrm{He}$ that is recognized to act as an inert element. Because of this, fusion reaction is clean and far superior to nuclear fission. 
Hot fusion spontaneously occurs at very high temperatures. Getting the necessary high temperature for hot fusion is still difficult and for that reason we must focus now on cold nuclear fusion. In order to induce cold fusion we need to accelerate the Deuterium nuclei in linear or circular accelerators. The proper energy of accelerated Deuterium nuclei should be well calibrated for a positive final yield of fusion reactions (to induce more nuclei merging than fission).

Electromagnetic fields needed to maintain the plasma (cold or warm), should be held (especially at cold fusion) to constrain more closely the nuclei.

We need to blast the fuel with accelerated Deuterium nuclei. The fuel will be made from heavy water and Lithium. The optimal proportion of Lithium is to be tested. In order to achieve a strong ionization of the fuel, it is compelling to keep fuel in the plasma state. In these conditions, instead of Deuterium atoms, Deuterium nuclei (positive ions) are produced, which can be accelerated by the electromagnetic fields.

\section{Conclusion}

Environmental protection through implementation of green energies is progressively becoming a daily reality. Numerous sources of green energy were introduced in recent years. Although this process initially started with difficulties, it finally resulted in an acceleration and implementation of new green energy technologies. Nonetheless, new major obstacles are emerging. The most worldwide difficult obstacle encountered, especially for wind and photovoltaic electric power plants, is the not regular and predictable green energy production. This study proposes solutions designed to solve this unpleasant aspect of irregular production of green energy. The basic idea refers to the construction of specially designed nuclear power plants acting as energy buffers. Nuclear power plants, indeed, may behave as proper energy buffers able to work to a minimum capacity when the green energy (i.e., wind power or PV) is steadily produced (namely, when the energy generated by the turbines or PV panels is at full constant capacity) but that can also run at progressively increased capacities when the wind or solar energy production reduces or stops.

Windmill farms are reliable, economical, sustainable, friendly and affordable.

Nuclear fission power plants have secured a new large amount of energy needed by the blue planet, however, the time of nuclear fusion power plants is approaching with quick steps.

The work get two major contributions: 1-propose to the achievement of an energy buffer using nuclear power plants (for the moment on nuclear fission); 2-shows some theoretical aspects important needed to carry out the reaction of the fusion.

\section{Acknowledgement}

This text was acknowledged and appreciated by Dr. Veturia CHIROIU Honorific member of Technical Sciences Academy of Romania (ASTR) PhD supervisor in Mechanical Engineering, Professor Guanying Chen Harbin Institute of Technology and SUNY Buffalo China, Associate Professor Aniello Riccio SECONDA UNIVERSITA' DEGLI STUDI DI NAPOLI Italy, Dr. (Ms.) Shweta Agarwala Senior Research Scientist at Singapore Center for 3D Printing Nanyang Technological University Singapore, whom we thanks and in this way.

\section{Author's Contributions}

All the authors contributed equally to prepare, develop and carry out this manuscript.

\section{Ethics}

This article is original. Authors declare that are not ethical issues that may arise after the publication of this manuscript.

\section{References}

De Ninno, A., A. Frattolillo, A. Rizzo, E. Del Giudice and G. Preparata, 2002. Experimental evidence of ${ }^{4} \mathrm{He}$ production in a cold fusion experiment. ENEA Technical Report.

Halliday, D. and R. Robert, 1966. Physics, Part II. 1st Edn., John Wiley and Sons, Inc., New York.

Le Quéré, C., R. Moriarty, R.M. Andrew, J.G. Canadell and S. Sitch et al., 2015. Global carbon budget 2015. Earth Syst. Sci. Data, 7: 349-396.

DOI: $10.5194 /$ essd-7-349-2015

Peters, G.P., S.J. Davis and R. Andrew, 2012. A synthesis of carbon in international trade. Biogeosciences, 9: 3247-3276. DOI: 10.5194/bg-9-3247-2012

Petrescu, F. and R. Petrescu, 2015. Wind farms. Alternative Energy Magazine.

Petrescu, F.I. and J.K. Calautit, 2016. About nano fusion and dynamic fusion. Am. J. Applied Sci., 13: 261-266. DOI: 10.3844/ajassp.2016.261.266

Petrescu, F.I. and R.V. Petrescu, 2011. Perspective Energetice Globale. 1st Edn., CreateSpace Independent Publishing Platform, ISBN-10: 146813082X, pp: 80.

Petrescu, F.I. and R.V. Petrescu, 2014. Nuclear green energy. Iraqi J. Applied Phys., 10: 3-14.

Petrescu, F.I., 2012. Cold nuclear fusion. Plasma Phys. Fusion Technol., 44: 100-100. 


\section{Nomenclature}

$\varepsilon_{0}=>$ the permissive constant (the permittivity)

$\varepsilon_{0}=8.85418 E-12\left[\frac{C^{2}}{N \cdot m^{2}}\right]$

$\mathrm{h}=>$ the Planck constant

$h=6.626 E-34[\mathrm{~J} \cdot \mathrm{s}]$

$\mathrm{q}=>$ electrical elementary load

for a deuteron $q_{1}=q_{2}=|e|=|-1.6021 E-19|[C]$

$\mathrm{c}=$ the light speed in vacuum

$c=2.997925 E 8\left[\frac{m}{s}\right]$

$m_{0}[\mathrm{~kg}]=>$ the rest mass of particle in movement

$\mathrm{m}_{0}$ proton $\quad 1.67262 \mathrm{E}-27[\mathrm{~kg}]$

$\mathrm{m}_{0}$ electron $\quad 9.11 \mathrm{E}-31[\mathrm{~kg}]$

$\mathrm{m}_{0}$ deuteron $\quad 3.34524 \mathrm{E}-27[\mathrm{~kg}]$ 